\title{
Out of hours care: a profile analysis of patients attending the emergency department and the general practitioner on call
}

\author{
Hilde Philips ${ }^{1 *}$, Roy Remmen ${ }^{1}$, Peter De Paepe ${ }^{2}$, Walter Buylaert ${ }^{2}$, Paul Van Royen ${ }^{1}$
}

\begin{abstract}
Background: Overuse of emergency departments (ED) is of concern in Western society and it is often referred to as 'inappropriate' use. This phenomenon may compromise efficient use of health care personnel, infrastructure and financial resources of the ED. To redirect patients, an extensive knowledge of the experiences and attitudes of patients and their choice behaviour is necessary. The aim of this study is to quantify the patients and socioeconomical determinants for choosing the general practitioner (GP) on call or the ED.

Methods: Data collection was conducted simultaneously in 4 large cities in Belgium. All patients who visited EDs or used the services of the GP on call during two weekends in January 2005 were enrolled in the study in a prospective manner. We used semi-structured questionnaires to interview patients from both services.

Results: 1611 patient contacts were suitable for further analysis. 640 patients visited the GP and 971 went to the ED. Determinants that associated with the choice of the ED are: being male, having visited the ED during the past 12 months at least once, speaking another language than Dutch or French, being of African (sub-Saharan as well as North African) nationality and no medical insurance. We also found that young men are more likely to seek help at the ED for minor trauma, compared to women.

Conclusions: Patients tend to seek help at the service they are acquainted with. Two populations that distinctively seek help at the ED for minor medical problems are people of foreign origin and men suffering minor trauma. Aiming at a redirection of patients, special attention should go to these patients. Informing them about the health services' specific tasks and the needlessness of technical examinations for minor trauma, might be a useful intervention.
\end{abstract}

\section{Background}

Overuse of emergency departments (ED) is of concern in Western society and it is often referred to as inappropriate' use [1-6]. Patients assess their medical problems with worries and interpretations in their own context and may decide to seek help independently from referral or triage systems $[7,8]$. Although there is some consensus of doctors and nurses concerning the perception of 'emergency', important differences were found between the perception of patients and clinical staff $[9,10]$. Patients' perceptions of an emergency do

\footnotetext{
* Correspondence: hilde.philips@ua.ac.be

'Department of Primary and Interdisciplinary Care of the University of Antwerp, Belgium (PICA-UA), General Practice, Universiteitsplein 1, Geb R, 3de verd., B-2610 Wilrijk, Belgium

Full list of author information is available at the end of the article
}

not necessarily correspond with clinical interpretations made by health care providers [11]. What is or is not an 'emergency' can lead to different interpretations of 'appropriate' and 'inappropriate use' of ED.

Inappropriate use may compromise efficient use of health care personnel, infrastructure and financial resources of the ED [12]. Inefficient use also threatens timely treatment of serious medical conditions at the ED $[13,14]$. The opinion to redirect patients, however, is hampered by the discrepancies in appreciation between consumers, health care providers and financial backers as to the value of primary and secondary care services. Therefore the top down approach alone is insufficient as a solution. An extensive knowledge of the experiences and attitudes of patients and their choice behaviour is necessary. Services must pay attention to this knowledge 
to align out of hours care to people's preferences, in order to attract patients to the most efficient service [15].

We therefore, in a prospective study, compared populations of patients during out-of-hours at both secondary care services (emergency departments, EDs) and primary care services (general practitioners (GPs) on call). The aim was to quantify the patients and socioeconomic determinants associated with choosing the GP on call or the ED. We also detailed reasons that patients mentioned for choosing a particular service.

\section{Methods}

Context

Belgian health care is characterized by free entrance to primary, secondary and tertiary care facilities. There is no gatekeeper role of general practitioners (GP) and no need for referral [16]. Physicians are most often paid on a 'fee for service' basis. Patients have obligatory medical insurance by which certain medical care is reimbursed. Out of pocket payment accounts for approximately $25 \%$ of health expenses [16]. For primary care, patients pay directly, while for secondary care, patients receive billings afterwards. At the time of the study co-payment systems at the ED were not compulsory and not in common use. Patients can be registered with a GP of their choice, but this is not obligatory to have access to all health care facilities. In Belgium, almost 99\% of the population is covered with compulsory health insurance [16,17].

Providing 24 hours coverage is a legal obligation of GPs in Belgium [18]. GPs organise out-of-hours care in rotation systems. This service is organised by local general practitioner organisations. In these small scale organisations, GPs on call usually work from their private practices. Most of the local GP organisations use a phone number which immediately leads to the out-ofhours care facility. Patients have to find out for themselves which GP is available and where the practice is located. Prior telephone contact is not necessary; patients can walk in without appointment. There is no telephone triage; no consultation over the telephone is performed. Patients can come to the doctor's practice or ask the GP for a home visit [16]. Since 2003, in some regions in Belgium, the first general practitioner cooperatives (GPC) emerged.

\section{Materials}

Data collection was conducted simultaneously in 4 large cities in Belgium (Antwerp, Ghent, Brussels, Charleroi). All patients who visited EDs or used the services of the GP on call during two weekends in January 2005 (Saturday 12 AM until Sunday 12 AM) were enrolled in the study in a prospective manner.

Directors of hospitals and primary care services were individually informed of the project and their participation was secured. The GPs on call and the services in the hospitals were regularly contacted by the principal investigator. Ethical approval was acquired for all services.

A semi-structured questionnaire was developed, based on literature, and piloted for this study. It comprised 6 domains and 39 questions. Senior medical students were trained to interview the patients at the various data collecting sites. They performed face-to-face interviews at the ED and telephone interviews with the GP patients after the doctor's visit. At the ED patients were asked to participate at the moment of entrance and data were collected immediately thereafter. As GP services were in many cities, and offered by more than one GP per region, we decided to collect data from these services by phoning immediately on the data of visit. GPs asked all patients whether they were willing to participate. If they agreed, the telephone number of the patient was registered in order to be contacted by the interviewer after the GP consultation.

For each patient the following data were collected about the consultation: demographic information (sex, age, postal code), date and hour of consultation, the Reason For Encounter (RFE), the diagnosis and whether or not subsequent hospitalisation was necessary. Also the manner by which they came to the medical service (self referral, physician's referral, ambulance, other) was registered. To assess the process of choice we also asked how they found the telephone number and address of the service, who made the decision to seek help at that service, what was their knowledge concerning the payment system, whether there had been earlier contacts with outof-hours services and whether they had considered looking for help elsewhere. At the end of the interview the socio-economic status (family, nationality, language, income/financial situation, insurance) was registered. Patients who refused to participate were only asked for their characteristics (age, sex) and the RFE. When possible we also assessed the doctor's diagnosis and whether the patient was hospitalised or not after the doctors' examination. Data of non-participants were only used to assess case-load but not for further analysis.

After data collection, the researchers used ICPC2 to recode RFE and diagnosis. The variable 'minor trauma' was collected by searching the data manually and adding the code A80 when trauma was mentioned in the RFE. When a A80 code in the RFE was combined with a S18 (skin lesion) or an ICPC2 code concerning contusions and abrasions in the diagnosis, we included the case as 'minor trauma'.

\section{Data collection and analysis}

Data were analysed using SPSS 16.0. We compared absolute numbers of contacts for each ICPC2 chapter 
between ED and GP contacts. Due to missing data concerning diagnosis in the data of Brussels and Charleroi, we restricted the descriptive analysis for the variables RFE and diagnosis to the data of Antwerp and Ghent.

We used uni-variant analysis with odds ratios (OR) and $95 \%$ confidence intervals $(\mathrm{CI})$ where applicable. Nominal variables were compared with chi $^{2}$-tests, whereas Mann Whitney tests were applied for the comparison of mean ages.

Binary logistic regression analysis with service choice (GP or ED) as the dependent variable, was used to compare patient and socio-economic determinants between both patient populations, computing odds ratios with their $95 \% \mathrm{CI}$. The choice of the determinants, relevant for this multivariate analysis was based on literature $[4,19,20]$.

\section{Ethical approval}

Ethical approval of this study was given by the Ethical Committees of the Universities of Antwerp, Ghent and Leuven: A04-77.

\section{Results}

\section{Descriptive}

A total of 1970 patients contacted one of the services and were eligible for inclusion at the four sites. 359 (18.2\%) patients refused to participate. Reasons for refusal were documented in 27 (0.07\%) cases: patient died $(\mathrm{n}=2)$, the patient is an unaccompanied child $(\mathrm{n}=19)$ or the patient was not able to participate $(n=6) .1611$ patient contacts were suitable for further analysis, 640 in the GP population and 971 ED users. Main patient characteristics are listed in table 1.

Refusal rate of study participation, was significantly lower in the GP visitors (GP: 113 refusals (15\%), ED: 246 refusals $(20 \%))$. The mean age (33.6 y, Standard Deviation (SD) 34.2) of the participants $(\mathrm{N}=1611)$ was not significantly different from the mean age of the non-participants $(\mathrm{N}=359)(38.0 \mathrm{y}, \mathrm{SD} 24.2)(\mathrm{P}>0.05)$.
Men were more likely to refuse participation than women did (refusals: male $\mathrm{N}=177$ (58.8\%), female $\mathrm{N}=$ $124(41.2 \%))(\mathrm{p}<0.01)$. The relative numbers of subsequently hospitalised patients were significantly higher in the nonparticipants group compared to those in the participants group (hospitalised non-participants $\mathrm{N}=64 /$ 225 (28.4\%), hospitalised participants $\mathrm{N}=206 / 1461$ $(14.1 \%))(\mathrm{p}<0.01)$. The mean age of the patients that visited the GP on call is 35.7 (SD 45.9) years, which is significantly higher than the population at the ED (32.2 y, SD 23.3) $(\mathrm{p}<0.05)$.

In the next part of this results chapter, we will focus only on the group of patients who participated $(\mathrm{n}=1611)$.

The item 'diagnosis' was missing in $49.4 \%$ of cases in the GP group $(\mathrm{N}=640)$. In the ED group only $3.8 \%$ of this data were missing $(\mathrm{N}=971)$. Therefore we limited the descriptive part on this specific item to the databases of Ghent and Antwerp, where registration of 'diagnosis' was performed as planned in the study design. Table 2 shows RFE and diagnosis chapters in both services. For the diagnosis, chapters L 'musculoskeletal' (21.6\%) and S 'skin' (17.3\%) were the most prominent at the ED services, while R 'respiratory' (36.8\%) and D 'digestive' (20.2\%) were most prominent at the GP services.

In the group of patients who decided to consult the GP $(\mathrm{N}=640), 54(8.4 \%)$ patients were not registered with a GP. In most cases the patient or a family member recommended calling the GP (93.2\%). In this group of patients $(\mathrm{N}=640), 105(16.4 \%)$ initially considered going to the ED but decided to call the GP. 185 (28.9\%) of the GP patients reported using the ED at least once during the past 12 months.

In the ED group ( $\mathrm{N}=971), 213(21.9 \%)$ patients were not registered with a GP. In $86.6 \%$ of the cases ( $\mathrm{n}=$ 841 ), the decision to go to the ED was taken by the patient or by a family member. In $8.0 \%$ of the cases $(n=$ 78) someone else gave the advice to visit the ED

Table 1 main patient characteristics at the GP services and the ED

\begin{tabular}{lll}
\hline & GP & ED \\
\hline Gender (\% men) & $289 / 638(45.3 \%)$ & $492 / 968(50.8 \%)$ \\
\hline Mean age & 35.7 years, SD 45.9 & 32.2 years, SD 23.3 \\
\hline Registered with a GP (\% yes) & $584 / 638(91.5 \%)$ & $754 / 967(78.0 \%)$ \\
\hline Used ED at least once during past 12 months (\% yes) & $185 / 63429.1 \%)$ & $379 / 960(39.5 \%)$ \\
\hline Employed (\% yes) & $354 / 622(56.9 \%)$ & $487 / 955(51.0 \%)$ \\
\hline First language Dutch or French (both national Belgian languages) (\% yes) & $580 / 639(90.8 \%)$ & $747 / 968(77.2 \%)$ \\
\hline Nationality: & & $642 / 968(66.3 \%)$ \\
$\quad$ - Belgian & $526 / 640(82.2 \%)$ & $129 / 968(13.3 \%)$ \\
$\quad$ - African & $20 / 640(3.1 \%)$ & $197 / 968(20.4 \%)$ \\
\hline
\end{tabular}


Table 2 Absolute numbers of patients visiting the ED or the GP with Reason For Encounter (RFE) and Diagnosis according to ICPC2 chapters (database of Ghent and Antwerp)

\begin{tabular}{|c|c|c|c|c|c|c|}
\hline \multirow[t]{2}{*}{ ICPC2 chapter } & \multicolumn{3}{|c|}{ RFE } & \multicolumn{3}{|c|}{ Diagnosis } \\
\hline & GP service & ED & Total & GP service & ED & Total \\
\hline Missing & 2 & 1 & 3 & 14 & 2 & 16 \\
\hline General and unspecified & 96 & 83 & 179 & 31 & 52 & 83 \\
\hline Blood, blood forming organs & 1 & 0 & 1 & 1 & 4 & 5 \\
\hline Digestive & 68 & 62 & 130 & 68 & 60 & 128 \\
\hline Eye & 3 & 13 & 16 & 1 & 11 & 12 \\
\hline Ear & 9 & 5 & 14 & 13 & 4 & 17 \\
\hline Circulatory & 6 & 8 & 14 & 7 & 25 & 32 \\
\hline Musculoskeletal & 33 & 119 & 152 & 27 & 106 & 133 \\
\hline Neurological & 19 & 26 & 45 & 10 & 17 & 27 \\
\hline Psychological & 6 & 17 & 23 & 7 & 19 & 26 \\
\hline Respiratory & 72 & 47 & 119 & 124 & 56 & 180 \\
\hline Skin & 17 & 58 & 75 & 21 & 78 & 99 \\
\hline Endocrine, metabolic, nutrition & 0 & 0 & 0 & 0 & 3 & 3 \\
\hline Urological & 2 & 6 & 8 & 8 & 8 & 16 \\
\hline Pregnancy, child-bearing, family planning & 0 & 0 & 0 & 1 & 1 & 2 \\
\hline Female genital & 0 & 3 & 3 & 1 & 2 & 3 \\
\hline Male genital & 2 & 2 & 4 & 2 & 2 & 4 \\
\hline Social problems & 1 & 0 & 1 & 1 & 0 & 1 \\
\hline Total & 337 & 450 & 787 & 337 & 450 & 787 \\
\hline
\end{tabular}

(friends, neighbours, ...). Of this group (this question was answered by $\mathrm{N}=681), 86(12.6 \%)$ patients contacted the GP on call before going to the ED. The question of by whom they were referred to the ED was answered by 968 participants. In 618 cases (63.8\%) patients reported going to the ED on their own initiative. Other referral possibilities were: referred by their own family physician ( $\mathrm{n}=67,6.9 \%$ ), by the GP on call ( $\mathrm{n}=57,5.9 \%)$ or by a specialist doctor $(\mathrm{n}=48,5.0 \%)$. 126 were brought in by ambulance $(\mathrm{n}=99,10.2 \%)$ or police ( $\mathrm{n}=27,2.8 \%$ ).

On $\mathrm{Chi}^{2}$ analysis, we found that men are more likely to seek help at the ED for minor trauma, compared to women. $(\mathrm{OR}=1.329$, 95\% CI: 1.010-1.749) This difference is not significant at the GP services $(\mathrm{OR}=0.820$, 95\% CI: 0.507-1.327).

People at the ED were asked why they decided to seek help at the ED. In order of absolute numbers the reasons are shown in table 5 .

Table $3 \mathrm{Chi}^{2}$ analysis of trauma and non-trauma related RFE between men and women at the ED

\begin{tabular}{llll}
\hline ED $(\mathbf{N}=\mathbf{9 7 1})$ & Trauma & Non-trauma & \\
\hline Male & 165 & 327 & 492 \\
\hline Female & 131 & 345 & 476 \\
\hline Total & 296 & 672 & 968
\end{tabular}

$\mathrm{Chi}^{2}=4.124, \mathrm{p}=0.0423$

$\mathrm{OR}=1.329,95 \% \mathrm{Cl}: 1.010-1.749$
Of the 971 patients who visited the ED in our study, 379 (39.3\%) had used the ED during the past 12 months at least once, $48(4.9 \%)$ of them more than 3 times.

\section{GP or ED? A binary logistic regression analysis}

We used binary logistic regression analysis with the use of the service (ED or GP) as dependent variable (GP being the reference category). Our best fitting model is described in table 6. We used 11 independent variables in the equation and six of them contributed significantly. Determinants that steered the choice in favour of the GP on call are: being female, having a family doctor and speaking Dutch or French (both national languages in Belgium). Determinants that advanced the choice for the ED are: being male, having visited the ED during the past 12 months at least once, speaking another language than Dutch or French, being of African (subSaharan as well as North African) nationality and lack of any medical insurance. Age, educational level and

Table $4 \mathrm{Chi}^{2}$ analysis of trauma and non-trauma related RFE between men and women at the GP services

\begin{tabular}{llll}
\hline GP $(\mathbf{N}=\mathbf{6 4 0})$ & Trauma & Non-trauma & \\
\hline Male & 32 & 257 & 289 \\
\hline Female & 46 & 303 & 349 \\
\hline Total & 78 & 560 & 638 \\
\hline
\end{tabular}

$\mathrm{Chi}^{2}=0.655, \mathrm{p}=0.4185$

$\mathrm{OR}=0.820,95 \% \mathrm{Cl}: 0.507-1.327$ 
Table 5 Reasons for seeking help at the ED

\begin{tabular}{lc}
\hline \multicolumn{1}{c}{ Question: 'Why did you decide to seek help at the ED?' (more answers possible) $(\mathbf{N}=\mathbf{9 7 1 )}$} \\
\hline Reason & Absolute number of patients who checked the box (\%) \\
\hline Accessibility & $140(14.4 \%)$ \\
\hline Competence of personnel & $110(11.3 \%)$ \\
\hline Proximity & $107(11.0 \%)$ \\
\hline Open 24/7 & $88(9.1 \%)$ \\
\hline No knowledge of GP on call & $70(7.2 \%)$ \\
\hline Family doctor not available & $50(5.1 \%)$ \\
\hline No need for an appointment & $39(4.0 \%)$ \\
\hline Not wanting to disturb the GP on call & $26(2.6 \%)$ \\
\hline No need for immediate payment & $10(1.0 \%)$ \\
\hline
\end{tabular}

employment were not significant in this regression model.

'Income' (missing in $49.7 \%$ of cases) and 'family situation' were entered into the model but did not change the results significantly. Adding interaction terms 'nationality*language' or 'age"sex' did not ameliorate the model significantly either.

\section{Discussion and Conclusions}

In this prospective study we compared profiles of 1611 patients at EDs and GP out-of-hours services in urban areas. Determinants for choosing a service were gender, having a family GP, having used the ED at least once during the past 12 months, language, nationality and having medical insurance.

According to table 2, musculoskeletal problems were the most frequent RFE and diagnoses at the ED. When keeping in mind that most RFE and diagnoses in ICPC-chapter S 'skin' are wounds or other traumatic skin lesions, we count $14.8 \%$ in the RFE at the GP and $39.3 \%$ at the ED that can be categorised as '(minor) trauma'. The same results are found for diagnoses: respectively $14.2 \%$ and $40.9 \%$.

Table 6 OR with $95 \% \mathrm{Cl}$ of independent variables in the equation with the choice for ED or GP as dependent variable (GP is the reference category, an OR $>1$ is in favour of the ED)

\begin{tabular}{|c|c|c|c|c|}
\hline & \multirow[t]{2}{*}{$P$ value } & \multirow[t]{2}{*}{ OR } & \multicolumn{2}{|c|}{$95,0 \%$ C.I. for OR } \\
\hline & & & Lower & Upper \\
\hline Sex male (female) & 0,049 & 1,249 & 1,001 & 1,559 \\
\hline Not registered with GP (Yes) & 0,000 & 2,696 & 1,856 & 3,916 \\
\hline Did not visit the ED past 12 months (Yes) & 0,001 & 0,675 & 0,533 & 0,855 \\
\hline Education: No diploma or primary school & 0,064 & & & \\
\hline Secondary school & 0,870 & 0,972 & 0,691 & 1,367 \\
\hline University or High school & 0,098 & 0,726 & 0,496 & 1,061 \\
\hline Age category (> 60 y) & 0,339 & & & \\
\hline $0-14 y$ & 0,918 & 1,021 & 0,693 & 1,503 \\
\hline $15-59 y$ & 0,283 & 1,211 & 0,854 & 1,716 \\
\hline Language (other than Dutch/French) & 0,006 & & & \\
\hline $\begin{array}{ll}\text { French } \\
\text { F }\end{array}$ & 0,001 & 0,491 & 0,317 & 0,761 \\
\hline Dutch & 0,007 & 0,522 & 0,326 & 0,836 \\
\hline Unemployed (Employed) & 0,844 & 0,973 & 0,744 & 1,274 \\
\hline Nationality (Belgian) & 0,000 & & & \\
\hline African Sub-Saharan & 0,008 & 3,726 & 1,400 & 9,914 \\
\hline North African & 0,001 & 2,885 & 1,513 & 5,501 \\
\hline Turkish & 0,164 & 1,891 & 0,771 & 4,638 \\
\hline Other nationalities & 0,436 & 0,859 & 0,585 & 1,261 \\
\hline No medical insurance (Yes) & 0,032 & 3,231 & 1,106 & 9,442 \\
\hline Constant & 0,000 & 10,859 & & \\
\hline
\end{tabular}

(Significant determinants are in bold) 


\section{Limitations of the study}

Some limitations of this study need to be addressed. We had to deal with the absence of strict catchment areas of both ED and GPs on call. Due to health service organisation in Belgium, people can seek help wherever they choose. As the areas are not well defined, numbers of GP contacts and contacts at the ED do not necessarily cover all patients seeking urgent care and are not necessarily adding up to one hundred percent of medical consumption. For this reason we have to be careful in our conclusions concerning socio-economic minority groups at the ED, which may have come from the broader catchment areas, and this may lead to over interpretation of this particular group of patients.

We lacked information on diagnosis in approximately half of the GP cases, due to under-registration of these data in Charleroi and Brussels. Nevertheless, we compared our results to other studies and found very similar results in studies in France, Sweden and The Netherlands, therefore we presume satisfying validity of our data [21-24].

We managed to obtain information on the income of patients in $50.3 \%$ of all cases. Including this variable in the binary logistic analysis leads to a less valid model and was therefore omitted. Because we assume that income and other socio-economic factors influence the patient's choice, it was rather unfortunate to have missing data on this item. In former research socio-economic factors have variable influence on choice behaviour, therefore it would have been very interesting to make conclusions about those items in this setting [25-27]. Future research using 'Geographic Information Systems (GIS)' describing socio-economic factors regionally, might elicit its role on choice behaviour [28-30].

As severity of the medical problem was not included in the questionnaire, we have to take into account that we may not compare the reasons for seeking help at either one service in a valid way, for severity is a confounding factor. We may not conclude on 'appropriate' or 'inappropriate' use based on these findings, neither was this the scope of this study. We missed data on income. For this reason we intend to perform a new study in a qualitative design, in which it is more feasible to assess income and other socio-economic determinants.

\section{Findings}

Men are more likely to seek help at the ED, often with ICPC codes relating to minor trauma (OR for male patients seeking help for 'minor trauma' versus female patients: OR $=1.329,95 \%$ CI: $1.010-1.749$ ). This confirms results of former research in which specifically young men rather seek help at the ED for minor trauma, suggesting that they appear to link their problem to technical examinations [31]. The most frequently mentioned reasons for choosing the ED are similar to findings in a questionnaire study in the Netherlands [32]. As the results of our study are similar, this indicates that this group is relatively free to choose, whether the GP appears to take the role of a gatekeeper or not.

People who used the ED during the past 12 months tend to return to the ED, whereas people who being registered with a GP, tend to seek help in primary care during out of hours. This confirms that people tend to choose the service which they are already acquainted with, as we have shown in a questionnaire study in the general public $[2,31,33-35]$. On the other hand, as we did not ask about the seriousness of the medical problem, another possible explanation could be that patients, who have visited the ED during the past 12 months, have more serious illnesses than other patients or suffer complications of former and/or chronic illnesses. Until now literature describes a 'returning behaviour' to the service patients know, further research has to take the seriousness of the complaint and patients history into account, to clarify its role in the choice behaviour of the patient.

Patients of foreign nationality presented themselves significantly more at the ED, hence bypassing the GP services. Cultural identity has been suggested as one indicator for different behaviour in the health system [26]. As those patients are acquainted to the healthcare system of their country of origin, they have less knowledge about the accessibility and organisation of out-of-hours services in other countries. Therefore, one can imagine that the GP services are, due to their structure, not accessible enough, as information of the services is not communicated in their language. Different types of organisation exist; in some regions GPs organize out of hours services at GP cooperatives, whereas other regions switch every weekend between GPs on call in a certain sequence. Perhaps the GPs, who work from their private practice, are sometimes difficult to locate or harder to reach.

Although financial aspects are not significant in our model, for this part of the community they might be more critical. The fee for service at the GP service and direct payment, might act as a patient selector [36]. This finding needs further investigation to explore reasons for this phenomenon. A qualitative approach can be used to explore how this specific population can be reached and how health care can be organised to minimize disparities.

In our setting $39.7 \%$ of all enrolled patients used the GP out of hours care and $60.3 \%$ the ED. Of all ED users $63.8 \%$ went to the ED without any referral. These figures might be subject to the health system. In other West European countries e.g. the Netherlands, where GPs are gate keepers and patients cannot easily attend a 
medical service without referral or telephone contact this percentage of direct ED referral is $43 \%[32,37]$. Compared to research similar to ours, in The Netherlands and the UK, the number of ED visitors is much higher in Belgium than it is elsewhere [32]. Another explanation for this phenomenon could be the lack of any kind of telephone triage as it exists in other countries. In Belgium patients not only have free choice of medical services, but also free access. There is no need for any telephone contact before entering care facilities. This excludes steering choice behaviour by telephone triage in the current health care system in Belgium $[38,39]$. Implementation of triage systems in the future and research as to whether this might be a solution to redirect patients is therefore necessary.

One critical determinant is whether the patient has medical insurance [27]. Also in our study, people who do not have any medical insurance tend to go to the ED rather than to the GP. This finding could be explained by the current situation in this country where patients at the ED do not pay immediately and receive an invoice later on, while patients who go and see the GP need to pay directly. Studying socio-economic influences requires specific research, focusing on those regions where different minority groups are found. More research needs to be done concerning the influence of socio-economic factors as a driver for patient choice.

\section{Conclusion}

In this, and in another study made by our group, we found that, in general, patients prefer the type of out-ofhours service that they know and have experienced [31]. A large proportion of patients at the ED do report having a GP, thus encouraging people to have a GP would probably not directly influence behaviour during out of hours. Two populations that distinctively seek help at the ED for minor medical problems are people of foreign origin and young men suffering minor trauma. Therefore, taking care of minorities in society by informing them about the possibilities of medical services could help to reallocate patients to the appropriate service. Also informing young people about the needlessness of technical examinations for most injuries and the availability of GPs during out-of-hours, could redirect patient streams, without diminishing quality of care. More research needs to be done concerning the influence of socio-economic factors as a driver for patient choice.

\section{Acknowledgements}

We thank all researchers who had a major contribution in this study, especially Catherine Gourbin MD, Didier du Boullay, MD, Reginald Moreels, MD, Laurence Kohn and Mark Leys.
We especially address special acknowledgements to the directors and staff of all participating hospitals and General Practitioner associations: Hôpital Civil de Charleroi, Hôpital Saint-Jean (Brussels), Hôpital Saint-Pierre (Brussels), A.Z. Sint-Lucas (Ghent), A.Z. Maria Middelares (Ghent), Universitair Ziekenhuis Gent (Ghent), A.Z. Jan Palfijn (Ghent), A.Z. Sint Erasmus (Antwerp), A.Z. Stuivenberg (Antwerp), Allo Santé (Charleroi), SOS Médecins (Brussels), Centrale Huisartsenwachtdienst van de region Gent, Centrale

Huisartsenwachtdienst van de regio Antwerpen, Huisartsenwachtpost Deurne-Borgerhout (Antwerp).

\section{Author details}

${ }^{1}$ Department of Primary and Interdisciplinary Care of the University of Antwerp, Belgium (PICA-UA), General Practice, Universiteitsplein 1, Geb R, 3de verd., B-2610 Wilrijk, Belgium. ${ }^{2}$ Department of Emergency Medicine, Ghent University Hospital, De Pintelaan 185, B-9000 Ghent, Belgium.

\section{Authors' contributions}

$\mathrm{PH}$ contributed to the study design, data gathering, analysis and writing the text. RR contributed to the study design, data gathering and reviewing the text. DPP contributed to the study design, data gathering and reviewing the text. BW contributed to the study design, data gathering and reviewing the text. VRP contributed to the study design and in reviewing the text. All authors read and approved the final manuscript.

\section{Competing interests}

The authors declare that they have no competing interests.

Received: 26 May 2010 Accepted: 15 November 2010 Published: 15 November 2010

\section{References}

1. Sempere-Selva $T$, et al: Inappropriate use of an accident and emergency department: magnitude, associated factors, and reasons-an approach with explicit criteria. Annals of Emergency Medicine 2001, 37(6):568-79, see comment.

2. Martin A, et al: 'Inappropriate' attendance at an accident and emergency department by adults registered in local general practices: how is it related to their use of primary care? Journal of Health Services \& Research Policy 2002, 7(3):160-5.

3. Lee $A$, et al: How to minimize inappropriate utilization of Accident and Emergency Departments: improve the validity of classifying the general practice cases amongst the A\&E attendees. Health Policy 2003, 66(2):159-68.

4. Sanders J: A review of health professional attitudes and patient perceptions on 'inappropriate' accident and emergency attendances. The implications for current minor injury service provision in England and Wales. Journal of Advanced Nursing 2000, 31(5):1097-105.

5. Lee $A$, Lee $A$ : The need for integrated primary health care to enhance the effectiveness of health services. Asia-Pacific Journal of Public Health 2003, 15(1):62-7.

6. Liggins K: Inappropriate attendance at accident and emergency departments: a literature review. Journal of Advanced Nursing 1993, 18(7):1141-5.

7. Gill JM, C.L.t Reese, Diamond JJ: Disagreement among health care professionals about the urgent care needs of emergency department patients. Annals of Emergency Medicine 1996, 28(5):474-9, see comment.

8. Wolcott BW: What is an emergency? Depends on whom you ask. JACEP 1979, 8(6):241-3

9. Kelly $L$, Birtwhistle R: Is this problem urgent? Attitudes in a community hospital emergency room. Canadian Family Physician 1993, 39:1345-52.

10. Lowe RA, Bindman $A B$ : Judging who needs emergency department care: a prerequisite for policy-making. American Journal of Emergency Medicine 1997, 15(2):133-6.

11. Callen $\mathrm{J}$, et al: Emergency department use in a rural Australian setting: are the factors prompting attendance appropriate? Australian Health Review 2008, 32(4):710-20.

12. Carret MLV, Fassa AG, Kawachi I: Demand for emergency health service: factors associated with inappropriate use. BMC Health Services Research 2007, 7:131. 
13. Bernstein $S L$, et al: The effect of emergency department crowding on clinically oriented outcomes. Academic Emergency Medicine 2009, 16(1):1-10.

14. Vieth $T L$, Rhodes $K V$ : The effect of crowding on access and quality in an academic ED. American Journal of Emergency Medicine 2006, 24(7):787-94.

15. Gerard $\mathrm{K}$, et al: Reviewing emergency care systems 2 : measuring patient preferences using a discrete choice experiment. Emerg Med J 2004, 21(6):692-697.

16. European Observatory on Health Systems and Policies, Belgium, in Health systems in transition profile. WHO European Centre for Health Policy 2007, 194.

17. Corens D: Health system review: Belgium. Health Systems in Transition 2007, 9(2):1-172.

18. Remmen RRR, Teblick M, Demerre M, van Hemelen G, Janvier P: Huisartsenwachtposten in Vlaanderen: wat zijn de randvoorwaarden? Huisarts Nu 2007, 36(8):397-401.

19. Martin A, et al: 'Inappropriate' attendance at an accident and emergency department by adults registered in local general practices: how is it related to their use of primary care? Journal of Health Services \& Research Policy 2002, 7(3):160-5.

20. Mitchell T: Nonurgent Emergency Department visits: Whose definition? Ann Emerg Med 1994, 24:961-3.

21. Gentile $S$, et al: [Do non-urgent patients presenting to an emergency department agree with a reorientation towards an alternative care department?]. Revue d Epidemiologie et de Sante Publique 2009, 57(1):3-9.

22. Backman A.-S, et al: Characteristics of non-urgent patients. Cross-sectional study of emergency department and primary care patients. Scandinavian Journal of Primary Health Care 2008, 26(3):181-7.

23. Giesen $P$, et al: Patients either contacting a general practice cooperative or accident and emergency department out of hours: a comparison. Emergency Medicine Journal 2006, 23(9):731-4.

24. van Uden CJT, et al: The impact of a primary care physician cooperative on the caseload of an emergency department: the Maastricht integrated out-of-hours service. Journal of General Internal Medicine 2005, 20(7):612-7.

25. Green J, Dale J: Primary care in accident and emergency and general practice: a comparison. Social Science \& Medicine 1992, 35(8):987-95.

26. Padela Al, et al: Emergency medical practice: advancing cultural competence and reducing health care disparities. Academic Emergency Medicine 2009, 16(1):69-75.

27. Brim C: A descriptive analysis of the non-urgent use of emergency departments. Nurse Researcher 2008, 15(3):72-88

28. Graves BA: Integrative literature review: a review of literature related to geographical information systems, healthcare access, and health outcomes. Perspectives in Health Information Management 2008, 5:11.

29. Lee JE, et al: Utilization of the emergency room: impact of geographic distance. Geospatial Health 2007, 1(2):243-53.

30. Benigeri M: Geographic information systems (GIS) in the health field: an opportunity to bridge the gap between researchers and administrators. In Canadian Journal of Public Health. Volume 98. Revue Canadienne de Sante Publique; 2007:(Suppl 1):S74-6.

31. Philips $\mathrm{H}$, et al: Experience: the most critical factor in choosing afterhours medical care. Qual Saf Health Care 2010

32. Moll van Charante EP, ter Riet $G$, Bindels P: Self-referrals to the A\&E department during out-of-hours: Patients' motives and characteristics. Patient Education and Counseling 2008, 70(2):256-265.

33. Scott $A$, Watson MS, Ross S: Eliciting preferences of the community for out of hours care provided by general practitioners: a stated preference discrete choice experiment. Social Science \& Medicine 2003, 56(4):803-14.

34. Blank FSJ, et al: A descriptive study of heavy emergency department users at an academic emergency department reveals heavy ED users have better access to care than average users. Journal of Emergency Nursing 2005, 31(2):139-44.

35. Olsson M, Hansagi $\mathrm{H}$ : Repeated use of the emergency department: qualitative study of the patient's perspective. Emergency Medicine Journal 2001, 18(6):430-4.

36. Shipman C, et al: Patient-perceived benefits of and barriers to using outof-hours primary care centres. Family Practice 2001, 18(2):149-55.

37. Moll van Charante PEric, P.C.v.S.-O, Bindels JEPatrick: Out-of-hours demand for GP care and emergency services: patients' choices and referrals by general practitioners and ambulance services. BMC Family Practice 2007, $8(46)$
38. Twanmoh JR, et al: When overcrowding paralyzes an emergency department. Managed Care 2006, 15(6):54-9.

39. Moll van Charante EP, t.R.G, Drost S, van der Linden L, Klazinga NS, Bindels PJE: Nurse telephone triage in out-of hours GP practice: determinants of independent advice and return consultation. BMC Fam Pract 2006, 7(74).

\section{Pre-publication history}

The pre-publication history for this paper can be accessed here: http://www.biomedcentral.com/1471-2296/11/88/prepub

doi:10.1186/1471-2296-11-88

Cite this article as: Philips et al:: Out of hours care: a profile analysis of patients attending the emergency department and the general practitioner on call. BMC Family Practice 2010 11:88.

\section{Submit your next manuscript to BioMed Central and take full advantage of:}

- Convenient online submission

- Thorough peer review

- No space constraints or color figure charges

- Immediate publication on acceptance

- Inclusion in PubMed, CAS, Scopus and Google Scholar

- Research which is freely available for redistribution 